\title{
INDENTABILITY OF CONVENTIONAL AND NEGATIVE POISSON'S RATIO FOAMS
}

\author{
R. S. Lakes \\ K. Elms \\ Department of Biomedical Engineering \\ Department of Mechanical Engineering \\ Center for Laser Science and Engineering \\ University of lowa \\ lowa City, IA
}

\section{Abstract}

The indentation resistance of foams, both of conventional structure and of re-entrant structure giving rise to negative Poisson's ratio, is studied using holographic interferometry. In holographic indentation tests, re-entrant foams had higher yield strengths $\sigma_{y}$ and lower stiffness $E$ than conventional foams of the same original relative density. Calculated energy absorption for dynamic impact is considerably higher for re-entrant foam than conventional foam.

\section{Methods}

Specimens of copper foam were cut from a larger block using a high speed band saw. The highest speed setting was used to avoid any plastic deformation during the cutting process. The specimens are transformed to the re-entrant structure by triaxial compression. A vise was used to apply the compression, and two $1 / 2$ inch thick pieces of Plexiglass were placed on the faces of the vise in order to provide a smooth surface. The foam specimen was then placed in the vise and subjected to small compressions of approximately $1 \mathrm{~mm}$ each, in each of the three orthogonal directions. This compression sequence was repeated, on average 15-20 times, until the desired volumetric compression ratio (VCR) was reached.

The set-up of holographic equipment was designed to simplify interferometric fringe interpretation. In this set-up the light from the laser passes through a beam splitter and spatial filter which changes it from a concentrated beam to diverging light. The light then passes through two lenses to become collimated. A large glass plate acts as a beam splitter, and a portion of the light is reflected onto the object, becoming the object light. The remainder of the light passes through the plate and becomes the reference light. The object light illuminates the object and is reflected back through the beam splitter plate and onto the film. The reference light is reflected off another glass plate, which acts as a mirror, and onto the film. The observer views the reconstructed image by gazing from behind the film in the direction of the object.

The actual object consists of a foam sample mounted on a platform with cyanoacrylate glue. The indenting force is applied to the foam via a pivot device. The arm of the pivot has a platform at one end to hold the weights, and an indenter at the midpoint of the arm. The tip of the indenter contacts the surface of the foam sample to cause the indenting force. The Boussinesq solution was used for analysis. Because only the sides of the foam sample can be seen in this configuration, a tilted mirror was added so that the top surface could be viewed. The top surface is the plane of applied indenting force, and interference fringes of greatest importance will be visible in the mirror. Because the object light is perpendicular to the object, the unit vector of illumination is simplified, and the fringe interpretation relation reduces to $u_{z}=\frac{n \lambda}{2}$ in which $u_{z}$ is the out-of-plane displacement. The wavelength $\lambda$ for red helium neon laser light is $632.8 \mathrm{~nm}$. These tests used double exposure holographic interferometry, with one exposure in a stressed state, and the other in an unstressed state. The film, Agfa 8E75 or 10E75, was 
sandwiched between two glass plates and loaded into the plate holder. Because the film has a curvature, it must be allowed to settle between the plates for approximately one minute.

The first exposure is made with weights on the platform of the pivot, so that the material is in the stressed state. After the exposure the weights were removed, and the second exposure was taken with the material in the unstressed state. Exposure times were 20 seconds each, with 20 seconds between exposures for removal of the weights. The film was then removed and developed with Kodak D19 Developer, followed by Kodak indicator stop bath. Extinction ratio of the hologram was measured, and the hologram was bleached with a potassium dichromate bleaching mixture. After drying, holograms were viewed.

If permanent yield has occurred in the material, it will be indicated by the presence of one or more interference fringes, encircling the region of yield or damage. Also studied is the size of the yielded region, and any patterns in its behavior. The indentation threshold tests were designed to determine plastic yield via holographic interferometry in copper foam samples subjected to an indenting load.

Tests were performed on three groups of copper foam samples. Both conventional and re-entrant samples were tested for 20 pore/inch foam with original relative density of 0.04 ; and 60 pore/inch foam, with original relative density of 0.08-0.09. A sample of re-entrant 40 pore/inch was tested; original relative density was 0.05 . In addition, one sample of conventional Rohacell polymethylacrylamide foam was tested; relative density was 0.092 .

The average applied stress was determined from the classical elasticity solution for indentation by a circular flat rigid punch.

\section{Results}

Figure 1 plots outer yield radius against stress for samples of 20 pore/inch conventional and re-entrant foam, with original relative density of 0.04 . The outer yield radius is the distance between the edge of the indenter and the circular interference fringe (there was only one fringe observed in these tests); the stress is the average bearing stress along the $z$ axis, or direction of applied load.

The results indicate two important findings. First, the re-entrant sample yields at a higher stress. Second, the area of damage, or yield radius, is smaller in the re-entrant foam than in the conventional foam for equal applied stresses, even though the re-entrant foam material is more compliant. For both materials the graphs show that there appears to be an upper limit to the size of the damage area, which follows an initial peak value. The yield stress for the conventional foam was $0.23 \mathrm{MPa}$ at a strain of 0.0021 . The peak yield radius was 5.0 $\mathrm{mm}$ with a limiting value of approximately $3.9 \mathrm{~mm}$. The re-entrant sample, with volumetric compression ratio (VCR) of 2.2 , had a yield stress of $0.36 \mathrm{MPa}$ at a strain of 0.0067 . This sample had a peak yield radius of $2.8 \mathrm{~mm}$ with a limiting value of roughly $2.2 \mathrm{~mm}$.

For these tests, the indenter remained in the same location on the surface of the material throughout the testing procedure. The curve for the re-entrant material in Fig. 1 shows the effects of strain hardening; yielding followed by no yield at higher stresses. An additional series of tests was performed on the re-entrant sample to investigate yield without the effects of strain hardening. Each time a hologram was made, the indenter was in a new location on the material's surface. The yield radii for the tests on the new locations are equal or nearly equal to the initial peak radius, and again there is a limiting value to the yield radius.

Two samples of 60 pore/inch copper foam with initial relative density of $0.08-0.09$ were tested, one re-entrant and one conventional. Figure 2 plots the outer yield radius against applied stress for both materials. Yielding of the conventional sample occurred at a stress of $0.51 \mathrm{MPa}$ and strain of 0.0030 . The limiting yield radius was roughly $1.3 \mathrm{~mm}$, with no initial peak radius. Holograms made at 0.98 and $1.03 \mathrm{MPa}$ showed two circular yield fringes; the radius of both inner fringes is $0.9 \mathrm{~mm}$. This sample also shows the effects of strain hardening, as indicated by the fluctuation in yield radius. 


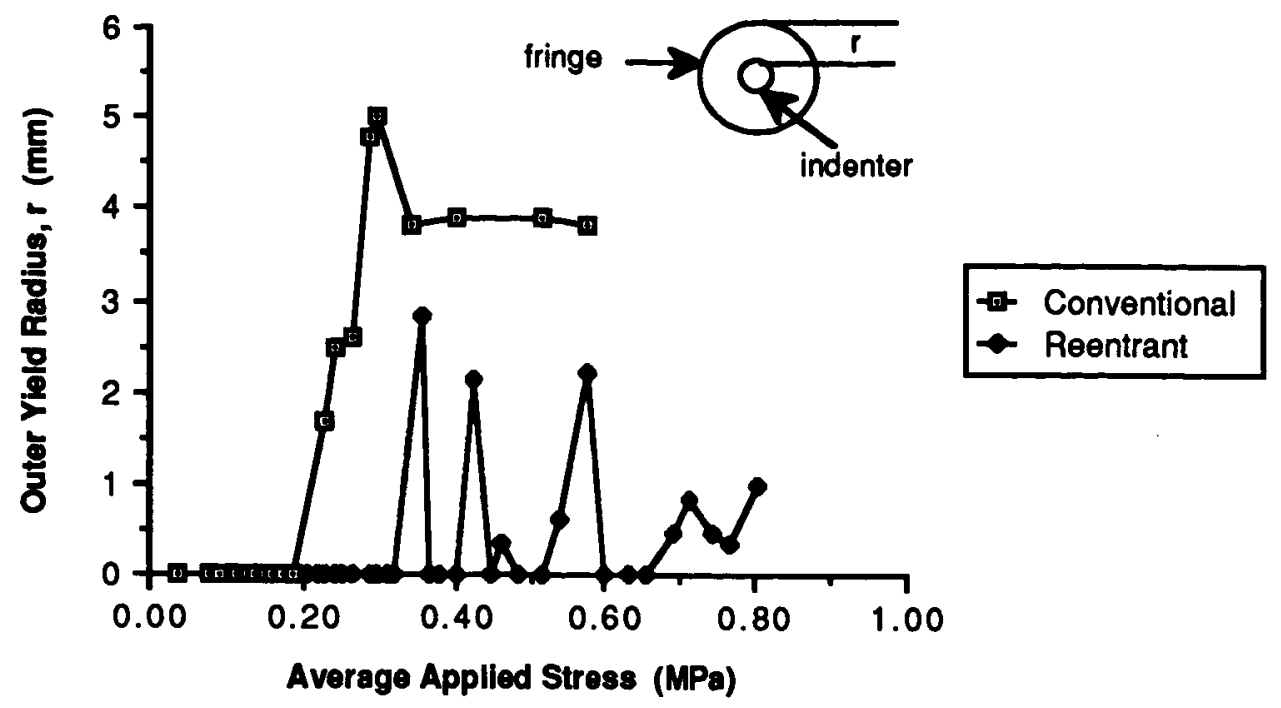

Figure 1. Outer yield radius vs. applied stress along the $z$ axis for both conventional and reentrant (permanent volumetric compression ratio $=2.2$ ) 20 pore/inch copper foams with initial relative density of 0.04 . Stress repetitively applied to same location.

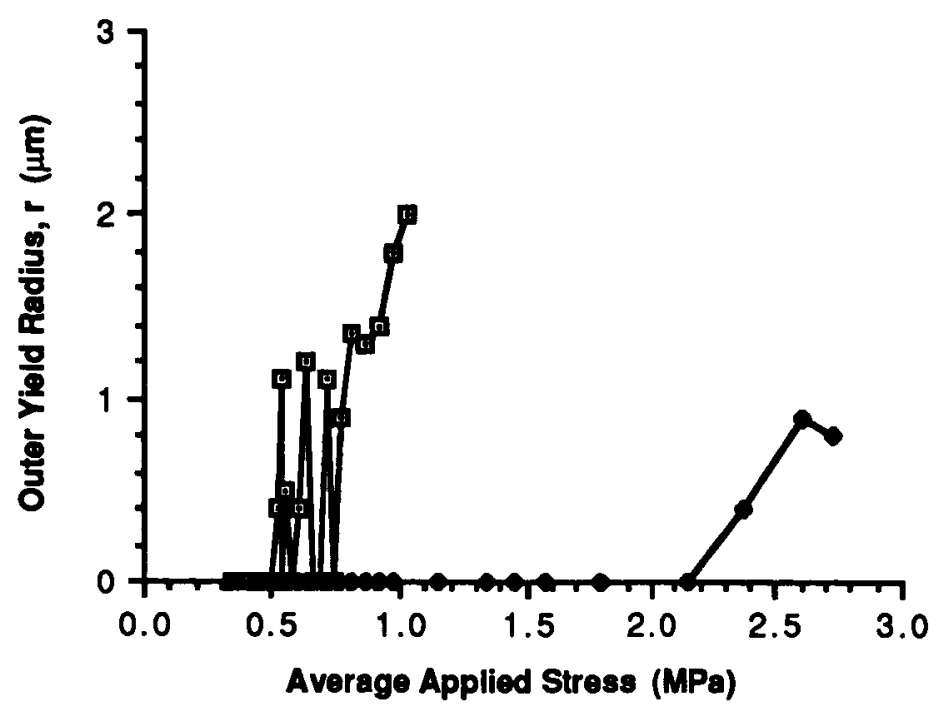

Figure 2. Outer yield radius vs. applied stress along the $z$ axis for both conventional and reentrant (permanent volumetric compression ratio $=2.3$ ) 60 pore/inch copper foams with initial relative density of 0.09 . Stress repetitively applied to same location.

The measurement of outer yield radius is not necessarily a true measure of actual yield. It is possible for the material to yield directly under the indenter by local crushing of the foam cells, and without lateral deformation or axial motion of material outside the region covered by the indenter. Therefore, actual yield may occur in the material without causing the formation of an interference fringe on the surface of the material. This behavior can be detected and 
measured from fringes that appear on the arm of the pivot device that is used to apply the indenting loads.

Conventional foam with an initial relative density of 0.09 yielded at a stress of 0.51 $\mathrm{MPa}$ and at a strain of 0.0030 . MTS compression tests on the same type of material showed a yield stress of $0.56 \mathrm{MPa}$ at a strain of 0.007 , as determined by the $0.2 \%$ offset method. Holographic bending tests on similar materials determined the yield stress to be $0.42 \mathrm{MPa}$ at a strain of 0.00047 [30]. The yield stress predicted by a structural model is $0.47 \mathrm{MPa}$; experimentally determined values for all methods are within the allowable range of $0.47 \pm$ $0.09 \mathrm{MPa}$.

Variation in measured values can be attributed to differing sensitivity of measurement techniques. Holography and shadow moire have greater sensitivity than the $0.2 \%$ offset method. Micro-yield can be observed holographically with a strain sensitivity of $10^{-5}$; the $0.2 \%$ offset method has a strain sensitivity of only $10^{-3}$. As a result, yield stresses measured holographically will be lower than by $0.2 \%$ offset, as will the yield strains.

The re-entrant sample with initial relative density of 0.08 and permanent volumetric compression ratio of 2.3 had a yield stress of $2.37 \mathrm{MPa}$ at a strain of 0.018 . Materials testing (MTS) tests on similar materials indicate yield at approximately $2.9 \mathrm{MPa}$ at a strain of 0.016 , determined by $0.2 \%$ offset. The yield stress for the outer yield fringe method is less due to the increased sensitivity of the method.

The re-entrant 20 and 40 pore/inch and the conventional 60 pore/inch copper foams had roughly similar final relative densities, $0.082,0.088$, and 0.086 respectively. As a result, a comparison of properties based on cell size can be made. The re-entrant 20 pore/inch sample had a yield stress of $0.33 \mathrm{MPa}$ at a strain of 0.0067 , its Young's modulus was $52 \mathrm{MPa}$, and the limiting outer yield radius was $2.2 \mathrm{~mm}$. The re-entrant 40 pore/inch sample had a yield stress of $0.86 \mathrm{MPa}$ at a strain of 0.015 ; its Young's modulus was $58 \mathrm{MPa}$ and it had a limiting outer yield radius of $1.4 \mathrm{~mm}$. The conventional sample had a yield stress of $0.51 \mathrm{MPa}$ at a strain of 0.0030 , its Young's modulus was $170 \mathrm{MPa}$, and the limiting outer yield radius was $1.8 \mathrm{~mm}$.

The 40 pore/inch re-entrant sample had the best yield strength and smallest damage region, although its stiffness was about one third the stiffness of the 60 pore/inch conventional foam with the same relative density. For dynamic loads, the compliance of the material can be beneficial. For a one-dimensional elastic buffer subjected to a dynamic impact force from a moving object, the maximum impact energy, $\mathrm{mv}^{2} / 2$ is in terms of core geometry,

$$
\frac{m v^{2}}{2}=\frac{1}{2} b c h\left(\frac{\sigma^{2} y}{E}\right)_{\operatorname{cor} \theta}
$$

To maximize the impact energy, a high $\sigma_{y}$ and a low $E$ are desirable. For the 20 and 40 pore/inch re-entrant foams and the 60 pore/inch conventional foam, which all have the same final relative density, the value of the $\sigma^{2} y / E$ term is $0.0021,0.013$, and 0.0015 , respectively. Therefore, the 40 pore/inch re-entrant sample provides both increased yield strength in the static case, and higher impact energy in the dynamic case.

Table 1

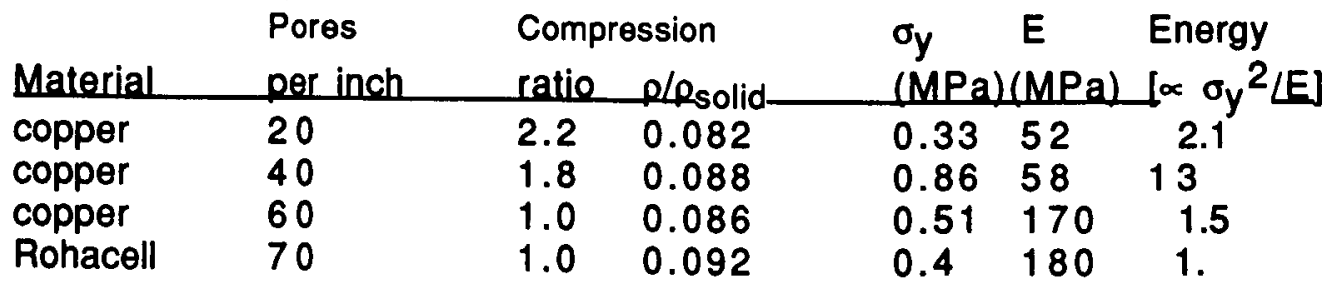




\section{Conclusions}

1. Foam core sandwich panels can be made more resistant to failure in certain modes if a re-entrant foam is used as the core material. This is based on both the increased yield strength and the negative value of the Poisson's ratio.

2. For materials with the same original relative density, re-entrant foams had a smaller outer yield radius (representing a damaged region) than conventional foams.

3. In holographic indentation tests, re-entrant foams had higher yield strengths $\sigma_{y}$ and lower stiffness $E$ than conventional foams of the same original relative density.

4. Calculated energy absorption for dynamic impact, $\propto \sigma_{y}^{2} / E$, was considerably higher for re-entrant foam. 